revista ANTHROPOLÓGICAS

Ano 21, 28(1):247-278, 2017

\title{
Des Origines Rituelles de l'Agriculture à l'Anthropocène. Réflexions sur un paradigme en formation aux multiples visages: l'agroécologie
}

\author{
Ludovic Aubin ${ }^{a}$ \\ Josefa Salete Barbosa Cavalcanti ${ }^{b}$
}

Cet article présente l'hypothèse d'une origine rituelle de l'agriculture. Il resitue l'agroécologie dans la longue histoire de l'agriculture à partir d'une perspective anthropologique, depuis ses origines (il y a environ 10000 ans) jusqu'à la période actuelle (que d'aucuns apellent l'Anthropocène) en passant par la rupture profonde que représente l'agriculture conventionnelle ou moderne. Nous mobiliserons et discuterons les notions de sacré et de désacralisation en considérant cette dernière et ses effets sur le monde comme la marque caractéristique de la modernité. Si le sacré contenait la violence de l'Homme (ou son hubris) dans les deux sens du verbe contenir, qu'advient-il lorsque ces dispositifs symboliques (rituels, interdits, obligations) tendent à perdre de leur prégnance?

Anthropologie; Sociologie de l'Agriculture; Agroécologie; Anthropocène.

Cet article trouve son origine dans une série de questions liées entre elles: pourquoi les paysans labourent-ils la terre? Qu'est-ce que le labour signifie en tant qu'intervention de l'homme sur la terre? L'agriculture archaïque inaugure-t-elle, il y a environ 10.000 ans, un

a Pesquisador em Pós-Poutorado (Capes/PNPD) no Programa de Pós-Graduação em Sociologia (UFPE). Membro do LAE-RURAL. Email: ludaubin@gmail.com.

b Professora da Pós-Graduação em Antropologia (UFPE) e da Pós-Graduação em Sociologia (UFPE). Email: saletecav@gmail.com. 
nouveau rapport de l'homme à la terre? L'événement agricole est-il si important qu'il déboucherait aujourd'hui sur une nouvelle ère géologique, l'Anthropocène, l'ère de l'Homme? Les réponses à ces questions ne sont pas évidentes. Elles nous obligent à revenir aux origines mêmes de l'agriculture pour comprendre ce que le labour signifie pour les premiers peuples agricoles et, en passant par l'agriculture moderne, à essayer de penser l'émergence de nouveaux paradigmes, comme l'agroécologie, capables de s'adapter au contexte global contemporain. Il s'agira de voir le chemin parcouru par les civilisations agricoles et comprendre ce qu'implique la désacralisation (Dupuy 2009:73) du rapport à la terre dans l'agriculture moderne et ce que cela signifie quant à l'augmentation considérable du pouvoir de l'agir de l'homme sur son monde.

Ce pouvoir considérable de l'Homme sur le monde ferait de ce dernier le principal agent de transformation de la Nature, ce qui amène Paul Crutzen (2000) à proposer l'usage du concept d'anthropocène pour désigner une nouvelle ère géologique qui succéderait ${ }^{1}$ à l'Holocène. Bien qu'encore en débat ${ }^{2}$, le concept d'Anthropocène permet une réflexion spéculative profonde sur les conditions historiques qui ont amené à cette situation globale nouvelle ainsi que sur le devenir des civilisations humaines et sur l'hybridation des sphères nature/culture.

Ainsi, dans la première partie nous reviendrons aux origines sacrificielles de l'agriculture (Girard 2004; Hocart 2005) en recourant à une approche comparée des mythes de fondation de celle-ci.

Dans la deuxième partie nous envisageons justement le formidable contraste que constitue l'approche moderne de l'agriculture par rapport à ses origines en envisageant le passage d'un modèle à l'autre sous l'angle de la notion de désacralisation.

Dans la dernière partie, nous considérons l'hypothèse de l'agroécologie comme une troisième voie en émergence: ni tributaire du sacré, ni prisonnière d'un rapport à la terre fondé sur la démesure et le rêve de maîtrise absolue propre à l'agriculture intensive contemporaine. 


\section{La méthode de recherches}

La première partie se base essentiellement sur une recherche documentaire et bibliographique comparative, à partir, principalement, de sources ethnographiques. La deuxième partie s'appuie essentiellement sur une approche théorique ainsi que sur les recherches de terrain réalisées dans Vale de São Francisco dans l'Etat de Pernambouc au Brésil. Les entretiens, témoignages et observations de la troisième partie proviennent essentiellement de trois sources: les travaux de groupe (mutirões en portugais) réalisés avec l'association Terra e Vida, des formations en permaculture et en agroforesterie que nous avons suivies entre 2012 et 2017 et des documents audiovisuels traitant du thème de la transition agroécologique.

L'agriculture ayant quelques 10.000 ans d'existence, le fait que depuis quelques siècles, voire quelques décennies, les sociétés contemporaines voient leurs agriculteurs devenir démographiquement et culturellement ultra-minoritaires, constitue un changement de nature anthropologique. C'est donc par la voie de l'anthropologie que nous commencerons par mettre en perspective les défis de l'agroécologie.

\section{Aux origines de l'agriculture: hypothèses en débat}

A la suite de plusieurs auteurs (Girard 1972, 1978, 2005; Hocart 2005), nous examinons dans cette partie l'idée d'une origine mythico-rituelle de l'agriculture. Dans cette perspective, ce sont les rites qui fournissent la matrice expérimentale au sein de laquelle peuvent se produire d'heureux hasards', des combinaisons bénéfiques, qui pourront être reprises et intégrées comme pratiques agraires. Cette hypothèse s'oppose à la conception utilitariste selon laquelle l'agriculture aurait été inventée pour des motifs immédiatement démographiques, économiques ou matériels ${ }^{3}$.

Quand l'on compare les mythes rendant compte, à leur manière, de l'invention de l'agriculture ou de la découverte des plants comestibles à la base de l'alimentation d'un groupe donné, on est 
frappé par la récurrence de certains thèmes et ce, quel que soit le foyer d'apparition concerné: le Moyen-Orient (Croissant Fertile), la Méso-Amérique, la Chine, le Nord de l'Amérique du Sud ou encore la Nouvelle-Guinée. La récurrence d'un même schéma fait dire à Mircea Eliade que:

"La conception de ces peuples, c'est que la plante alimentaire est le résultat d'un meurtre primordial. Un être divin a été tué, morcelé, et les morceaux de son corps ont donné naissance à des plantes inconnues jusqu'alors, surtout à des tubercules, qui depuis ce temps constituent la principale nourriture des humains" (Eliade 2006:138).

Ce dont les mythes rendent compte, en effet, c'est que l'acte (le meurtre) fondateur intègre subitement tous les éléments désordonnés du réel et leur donne sens les uns par rapport aux autres en les organisant. Il faut que l'acte soit d'une puissance considérable pour avoir cette capacité d'agencer en un tout signifiant des éléments épars ou désordonnés. Voici un mythe d'origine du manioc, tubercule si important dans le régime alimentaire des brésiliens: ${ }^{4}$

"Zatiamare et son épouse, Kôkôtêrô, avaient deux enfants, un garçon, Zôkôôiê, et une fille, Atiôlô. Le père aimait le garçon et méprisait la fille. Si elle l'appelait, il lui répondait en sifflant, mais jamais ne lui adressait la parole. Malheureuse, Atiôlô demanda à sa mère de l'enterrer vivante, sentant qu'elle pourrait ainsi être plus utile. Après avoir longtemps résisté à cette étrange demande, Kôkôtêrô finit par céder aux supplications de sa fille et l'enterra au milieu de l'enclos. Cependant, la fille ne put supporter la chaleur, et implora que sa mère l'emmenât plus loin vers le champ. Mais là elle ne sentait pas bien non plus. Une fois encore elle implora Kôkôterô qu'elle la déplaçât vers un autre trou, dans une clairière au milieu de la forêt. Là elle se sentit bien. Elle demanda alors à sa mère de partir en lui recommandant de ne pas tourner le regard quand elle crierait. Après longtemps, elle cria. Kôkôtêrô s'en revint rapidement. Elle vit alors, au lieu où elle avait enterré sa fille, un arbuste très haut, qui rapetissait à mesure qu'elle s'approchait. Elle s'occupa de la sépulture. Nettoya le sol. La petite plante se montra chaque fois plus vigoureuse. Plus tard, Kôkôtêrô arracha du sol la racine de la plante. C'était le manioc" (Brandenburger 1931:34-35). 
Dans ce mythe, le corps n'est pas démembré. En revanche, on peut l'interpréter comme un infanticide commis par la mère (qui cesse d'assumer son rôle de mère, protectrice de sa fille) pour le bénéfice du groupe ou, en considérant les inégalités de genre au sein du groupe, comme le meurtre de celle qui était méprisée par le groupe, et fait don de sa vie pour le groupe. C'est bien du corps du mort que naît la plante alimentaire ${ }^{5}$.

René Girard (1972) a fait de l'hypothèse du meurtre fondateur la clé de voûte de son anthropologie religieuse. Au terme d'une crise dont l'origine peut être endogène à la communauté (dissensions, rivalités entre frères ou entre proches de manière générale, jalousies, etc.) ou exogène (phénomène climatique), le désordre 's'empare' de celle-ci. L'ordre s'écroule. Il y a alors violence de tous contre tous, actuelle ou potentielle. La tendance spontanée des groupes humains est d'attribuer ce désordre à une différence contingente, certes, mais signifiante pour la communauté. Se met alors en place le mécanisme victimaire indissociable d'un système d'accusation (d'inceste, de sorcellerie, de mauvais oil, etc.). La communauté, ou un représentant légitime de celle-ci, tue (ou pousse à la mort) un de ses membres, celui-ci étant considéré comme responsable de la crise. La mort de la victime a alors deux types d'effets. Tout d'abord, elle 'résout' la crise ${ }^{6}$ en apaisant la communauté. C'est l'effet cathartique du mécanisme. Ensuite, du corps enterré surgissent les plants dont les produits et les sous-produits constitueront la base alimentaire de la communauté! La communauté interprétera cet événement comme extrêmement favorable et s'appliquera à répéter aussi minutieusement que possible le meurtre fondateur et les conditions qui l'ont précédé. Ce sera le rituel sacrificiel. Dans la perspective de la théorie girardienne, le sacrifice est l'immolation d'une victime ${ }^{7}$ polarisant sur elle les forces potentiellement destructrices mais se révélant productrices de paix et d'abondance par l'entremise, précisément, du sacrifice. Le sacrifice canalise la violence répandue dans la communauté sur une victime unique dont la mort est le dernier mot de la violence car la victime ap- 
partient à une catégorie d'êtres incapable de se venger. La victime est alors divinisée. Ce qu'elle génère comme bénéfice, seul un être divin, pensera-t-on, peut l'apporter! En cela le sacrifice est la répétition (imitation) du meurtre fondateur dont les effets pour la communauté ont été heureux. Quant au mythe, c'est l'histoire qui narre la séquence des événements (crise/rupture de l'ordre, création de l'ordre après le meurtre) racontée $d u$ point de vue de la communauté. Il s'agit donc d'une version des faits qui lui est favorable.

Reprenons l'expression d'Eliade 'un être divin a été tué' et tentons de la préciser. Nous pouvons dire maintenant, avec Girard, que l'être devient divin après le meurtre! C'est la violence du meurtre et ses effets fondateurs, réconciliateurs, bénéfiques (socialement et biologiquement) qui rendent la victime divine! Elle a des pouvoirs que nul homme pris isolément ne peut avoir ou maîtriser. Pour garantir la perpétuation des effets bénéfiques, il faudra répéter le geste fondateur qui fabrique des dieux. C'est le rituel sacrificiel. Avant Girard, l'ethnologue Grant Allen soutenait déjà cette thèse en affirmant que "les dieux sont des fabrications humaines par immolation de victimes" (1898:10-11) sans en avoir tiré les mêmes conclusions $^{8}$.

A travers ces exemples de mythes issus de régions du monde très diverses, nous avons voulu montrer la dimension éminemment rituelle des conditions d'apparition de la proto-agriculture. Comme pour toute activité importante mobilisant des forces cosmiques, physiques, biologiques et sociales, c'est à travers le rite que les hommes procèdent et parviennent à une maîtrise relative mais jamais garantie de ces forces. L'espèce humaine est donc, dans cette perspective, une espèce qui s'auto-domestique par l'entremise du rite. Autrement dit, "l'humanité est fille du religieux" (Girard 2004:172). Voyons donc à travers l'exemple de deux rites agraires les soins que les sociétés traditionnelles portent aux activités de labour, de semailles et de moissons. Cela éclaierera para contraste la singularité de l'agriculture conventionnelle et le défi de l'agroécologie. 


\section{Les rites de labour, de semailles et de moisson: de quoi le rite est-il la répétition?}

Au fondement du champ cultivé, il y aurait donc un mort issu d'un meurtre primordial, fondateur. Michel Serres note que la racine indo-européenne Pak qui signifie 'planter' fait aussi référence à la tombe comme signe premier, comme première inscription symbolique de l'homme (Serres 1993:56). Par extension, dit-il, les bornes qui marquent le champ pourraient bien être des tombes ${ }^{9}$. Grant Allen se demande:

"Quelle est l'unique circonstance où l'homme primitif a dû accomplir les préliminaires indispensables de l'agriculture: retourner le sol, ramener la terre à la surface, extirper les plantes sauvages, déposer dans le sol des graines et même de l'engrais? Les sauvages, répond $\mathrm{M}$. Grant Allen, ne creusent et ne retournent systématiquement la terre que dans un seul cas, quand il s'agit d'enterrer leurs morts $^{10 "}$ (Alviella 1898:10).

La tombe devient l'objet d'un culte, d'un soin sacré avec des offrandes, des libations qui enrichissent la terre, la rendent et la maintiennent fertile. A titre d'exemple, l'ethnologue rapporte qu'en Ecosse les paysannes continuent de verser du lait sur les fairy knowes ou tumuli préhistoriques: "sur ces tumuli, l'herbe y est plus verte, la terre plus fertile" (Alviella 1898:11).

Dans la Grèce antique, on pratiquait le rituel du pharmakos. En cas de crise sociale (épidémie, rumeurs de guerre avec des cités voisines) on choisissait un esclave entretenu à grands frais par la cité. Celui-ci était traîné dans la cité, molesté, invectivé ou encore fouetté puis immolé, incinéré et ses cendres étaient répandues sur les champs autour de la ville ${ }^{11}$.

Tous ces exemples, issus de régions et d'époques très diverses, laissent penser que, en-deçà de formes culturelles et rituelles locales et singulières, il existe des mécanismes anthropologiques morphogénétiques. C'est ce que nous avons commencé à voir en mentionnant l'hypothèse du meurtre fondateur. Poursuivons le raisonnement en 
nous appuyant sur l'exemple d'un rite agraire kabyle décrit et analysé par Jean Servier dans un article classique et un livre (Servier 1951 et 1962$)^{12}$.

Dans notre monde moderne où l'agriculteur est considéré comme un entrepreneur individuel 'comme un autre', il est important de rappeler que le labour, les semailles et les moissons étaient des activités communautaires dont les enjeux dépassaient largement la simple production alimentaire. Autrement dit, s'alimenter est plus qu'une nécessité physiologique, c'est le produit d'activités sociales complexes. L'agriculture mettait en branle et en jeu tout un ensemble de forces sociales et cosmiques dont l'agriculteur était l'un des opérateurs. Jean Servier rappelle que:

"Le labour est la pénétration dans le monde sacré, c'est-à-dire le monde non ouvré. Des précautions magiques devront être prises pour protéger le laboureur et ses compagnons les animaux, attelés sous le joug" (Servier 1951:184).

Le bœuf qui laboure le premier sillon sera, par exemple, sacrifié et son sang, répandu sur le champ. Le soc ayant servi à creuser le sillon sera déposé dans un sanctuaire, comme souillé par des forces potentiellement contagieuses et dangereuses car "la terre est le lieu où résident les esprits des morts” (Servier 1951:184). Ouvrir la terre, c'est donc déranger les divinités chtoniennes. En ouvrant le champ (en le profanant), l'homme et les bœufs devront se protéger contre l'excès de baraka (principe vital) de la terre inculte. Par ailleurs, lors des semailles, l'homme sera chaussé et coiffé, c'est-à-dire isolé magiquement des puissances d'en-haut et d'en-bas (Servier 1951: 185).

Enfin, après avoir ouvert le champ, on mettra du blé dans une tombe préparée longtemps à l'avance "afin qu'elle ne réclame pas un corps humain"13 (Alviella 1898:187). Sur la route de la maison au champ, le laboureur distribuera encore des fruits, figues sèches ou noix aux passants qu'il rencontrera afin d'écarter le mauvais oil (le regard d'envie) ou d'augmenter le nombre de participants au repas de communion afin que la baraka mise en ouvre soit plus grande (Ser- 
vier 1951:191). Enfin, la moisson s'accompagne de rites funéraires et se termine par la mise à mort rituelle de la dernière gerbe (Servier 1951:230).

Provenant en première instance du corps du mort dont la personne a été divinisée et dont les rituels tentent de maintenir vive la filiation, l'aliment est porteur de la substance divine. Ingérer l'aliment c'est ingérer un peu de la substance du dieu. Ainsi les aliments ne sont pas un élément passif du cycle de vie; ils sont des signifiants vitaux (Alviella 1898:116).

Jean-Pierre Dupuy dans son effort de formalisation de la théorie girardienne de la violence et du sacré a résumé en une formule concise le paradoxe fondamental de l'acte qui rend sacré, à savoir, le sacrifice. Le sacrifice (et les institutions qui en découlent - Girard 1972:122) contient la violence dans les deux sens du verbe contenir: il recourt à la violence (en immolant une victime) mais ce geste cathartique purifie (pour un temps au moins) les ferments de dissensions intra ou inter-communautaires ${ }^{14}$ et y fait donc barrage.

Si les institutions découlent du sacrifice et du rite qui en est la répétition (Hocart 2005), si l'agriculture est l'une de ces institutions, et si le sacrifice contient la violence dans les deux sens du verbe contenir, alors la critique moderne du sacrifice entraîne de proche en proche la perte de sa légitimité et de son efficacité ne permettant plus à la violence, ou en tout cas aux rivalités, de trouver d'exutoire, de marquer des limites et de produire de la culture.

Si, de plus, comme nous l'avons vu, le radical indo-européen Pak qui a donné planter (ainsi que pays, paysan, paysage, paix, païen, etc.) fait bien référence à la tombe comme signe premier, alors le paysan païen est l'être qui est relié à la terre par le sang de ses ancêtres et le paganisme constituait l'ensemble des religions qui honorait les morts, les ancêtres. Ainsi, le sacrifice était un élément essentiel des religions païennes. La christianisation des peuples païens, en interdisant la pratique des sacrifices, a résulté en une transformation profonde du rapport à la terre et au cosmos. On peut conjecturer que, pour l'Europe, 
17 siècles de christianisation ${ }^{15}$ puis de laïcisation du rapport à la terre ont achevé de désacraliser le rapport à celle-ci, la transformant en un simple support utilitaire, un substrat inerte ${ }^{16}$.

Cette dynamique historique profonde entraîne une déritualisation dans tous les secteurs de la vie sociale et, au premier chef, de l'agriculture, avec tout ce que cela signifie de perte de sens de la vie communautaire paysanne et de la profession d'agriculteur, d'isolement des agriculteurs, de perte de vitalité des aliments, de déracinement, etc. ${ }^{17}$

L'agriculture archaïque ou primitive n'est donc pas, dans les sociétés anciennes, un secteur d'activités semi-autonome contrairement à la manière dont elle est considérée dans la modernité. L'agriculture est par excellence cette activité d'intégration entre, d'une part, une dimension très humaine, requérant une coordination complexe de tâches et, d'autre part, des forces non-humaines (rythmes cosmiques, variations saisonnières, plantes, animaux, etc.).

Son 'invention' ne serait pas non plus le fruit d'une décision planifiée ou rationnelle au sens de la rationalité instrumentale, pour répondre à des nécessités économiques ou démographiques. L'argument de l'origine sacrificielle et rituelle de l'agriculture propose d'inverser le raisonnement de l'argument utilitariste. L'agriculture naîtrait de la découverte heureuse des effets du meurtre fondateur sur la communauté et sur la fertilité du sol: l'abondance (les plantes) et la paix apparaissent ainsi comme un don $d u$ mort. En cela, la plante qui en provient est sacrée car elle porte en elle la substance du dieu. Mais alors, pour répéter les effets heureux de l'événement fondateur, il sera rationnel (dans la rationalité religieuse) de recourir au rite sacrificiel. Par conséquent, ce sont les besoins du rite sacrificiel requérant de disposer facilement de victimes de rechange qui amènent à la domestication $^{18}$ et aux sous-produits de la domestication. La plante (et, finalement, la culture et, finalement, tout ce qui permet la vie) est donc 
vue comme un don du mort envers lequel la communauté nourrit un sentiment de crainte et de gratitude. Il nous semblait utile de faire ce détour et de formuler des hypothèses quant à l'origine de l'agriculture pour pouvoir mieux comprendre, par contraste, la singularité de l'agriculture moderne conventionnelle.

\section{Agriculture conventionnelle: en route pour l'Anthropocène}

Dans cette seconde partie nous envisageons l'agriculture conventionnelle comme participant d'un paradigme plus large, celui de la modernité. Nous réfléchissons ainsi sur la formidable rupture que représente le passage de l'agriculture traditionnelle, indexée au temps socio-cosmique, à l'agriculture conventionnelle. En s'auto-désignant moderne ou conventionnelle, l'agriculture issue de la 'Révolution verte' impose une doxa, réduisant les autres formes d'agriculture à des pratiques 'dépassées', 'rétrogrades', 'irresponsables' car 'incapables de nourrir le monde' ou de 'répondre à la demande croissante du marché'. Mais nous verrons comment, à l'instar d'autres secteurs des sociétés contemporaines, l'agriculture conventionnelle est devenue contreproductive par excès de puissance. En somme, l'agriculture conventionnelle s'inscrit dans une dynamique historique générale moderne qui mène aujourd'hui à l'anthropocène.

\section{a - Agriculture conventionnelle: rupture et continuité avec les agricultures archaïque et traditionnelle}

Selon le point de vue dominant des institutions et des acteurs contemporains, l'agriculture moderne intensive est envisagée comme une activité sectorielle dans une société en voie avancée ou complète de désacralisation et non plus comme une institution impliquant toutes les dimensions de la société.

En effet, si, comme on l'a vu, les institutions découlent du sacrifice et du rite qui en est la répétition (Hocart, 2005), alors la désacralisation correspond à la perte de légitimité et d'efficacité de ce qui com- 
pose le sacré, à savoir: les mythes, les rites (notamment sacrificiels), les interdits et les obligations (droits coutumiers du sol), les systèmes de différences (hiérarchies), le rapport au temps, etc. La désacralisation implique une perte de la peur du châtiment divin (ex. la Némésis) au moment de transgresser des limites sacrées (y compris temporelles) qui, par là-même, se diluent, disparaissent. Autrement dit, la désacralisation du monde crée les conditions socio-historiques permettant de considérer le sol et les produits de la terre comme de simples substrats et denrées utiles. La désacralisation implique une libération de la violence (l'excès de force) que le sacré jadis contenait.

Les travaux de Cavalcanti (2013 et 2014) et Bonanno \& Cavalcanti (2014) sur le pôle fruticole du Vale de San Francisco, dans l'Etat de Pernambouc au Brésil ont mis en lumière les transformations profondes des dimensions sociales et écologiques induites par une agriculture intégralement tournée vers le marché global: intensification des cadences de travail (au mépris parfois de la législation) et exploitation maximale des ressources naturelles locales. Les rythmes du travail et l'individualisation du contrôle électronique des travailleurs pour chacune de leurs tâches génèrent un double mouvement psychique: l'absence d'engagement affectif entre le travailleur et le produit de son travail et un stress accru dû à la crainte qu'une erreur (commise par exemple lors de l'étape de conditionnement des fruits) puisse être dénoncée par un consommateur à l'autre bout du monde, faire l'objet d'une plainte et être détectée avec exactitude (par l'identification électronique du poste de travail et des horaires). L'auteur montre enfin comment la focalisation de l'entrepreneur sur le marché et les exigences de celui-ci amènent à contrôler jusqu'à la vitesse de développement de la plante en jouant sur son rythme de croissance!

Car ces produits sont désormais distribués sur tout le globe, échangés en bourse et traités à la cadence des algorithmes du High Frequency Trading. Spéculation, vitesse, sont devenus les maîtres-mots des bourses mondiales, créant surabondance dans certaines régions du globe, rareté et pénurie dans d'autres avec une pression accrue sur 
les hommes et leurs milieux. Cette accélération sociale du temps est devenue, selon Hartmut Rosa (Rosa, 2013), un principe autonome et déterminant suffisamment puissant pour devenir, selon lui, la dimension caractéristique de la modernité tardive.

Nous avons proposé, pour notre part, à la suite de divers auteurs (Weber; 1917, Girard, 1996, Dupuy, 2009) de considérer la désacralisation comme le principe moteur de la modernité et de dire que l'accélération sociale du temps ne peut advenir qu'à condition que le temps soit devenue une simple ressource utile.

\section{b - Le sans-limite de l'agriculture conventionnelle: excès de puissance et contreproductivité}

En voulant fonder (et agir sur) leur monde à partir de leurs forces et de leur volonté propres, en autonomie, les sociétés modernes refusent toute hétéronomie. En croyant ne repérer au fondement du religieux que de l'arbitraire et de la superstition, celles-ci entendent se libérer du joug de toute limite. Ce faisant (et c'est l'expérience que les sociétés contemporaines sont en train de faire), en dépassant certains seuils critiques, les collectifs humains déclenchent dans la nature et dans le monde social des forces qui, par effet de composition, semblent acquérir une vie autonome recréant ainsi, paradoxalement, une forme d'hétéronomie (Dupuy 2009). La violence, le marché, le changement climatique semblent désormais échapper à tout contrôle, s'alimentant les uns les autres, alors même que, c'est incontestable, elles sont la conséquence (au moins partiellement) des actions humaines.

Il y a donc, dans la modernité, un double mouvement contradictoire, un chassé-croisé dans ce rapport humains/non-humains: un mouvement de perte de maîtrise à mesure que le pouvoir d'agir de l'homme sur son environnement s'accroît. C'est ce regard en chassé-croisé que nous aimerions poser sur l'agriculture, sur son évolution et sur les défis que l'agroécologie doit et devra relever.

Les agricultures d'aujourd'hui n'évoluent plus, en effet, dans le même monde que celui de Justus Von Liebig (1803-1873) pionnier 
de l'agriculture industrielle. Elles évoluent dans un monde modifié et saturé par les effets de la première phase de la modernité. La phase actuelle, que Beck appelle modernité réflexive (Beck 1986), est une phase historique de saturation où l'on évolue dans (et agit sur) un monde déjà transformé lors de (et par) la première modernité. Dire que, dans la modernité réflexive, il y a saturation revient à dire qu'il n'y a plus d'extériorité radicale susceptible d'accueillir le 'trop-plein' des sociétés contemporaines (trop-plein de violence, de pollution, etc.). Il nous faut donc penser une agriculture adaptée à notre phase historique dans laquelle il devient impossible de continuer à externaliser les coûts et la pollution qui viennent, par effet boomerang, affecter toute la société.

La question agricole sous l'angle anthropologique est donc paradigmatique du rapport de l'homme à son monde dans l'anthropocène. C'est au moment précis où le rêve de maitrise de l'homme sur son environnement atteint un niveau inédit dans l'histoire que les rapports les plus alarmants émanant d'institutions des plus officielles nous indiquent que des seuils critiques sont en passe d'être franchis ou ont déjà été franchis ${ }^{19}$. Or, au-delà de ces seuils, les systèmes affectés entrent dans une dynamique de comportements qui cesse d'être prévisible. C'est l'impossibilité de maîtriser les événements qui devient alors la norme. Il faut penser davantage ce paradoxe car il nous permet d'identifier une différence essentielle entre l'agroécologie et l'agriculture moderne intensive.

Le paradigme agronomique dominant, dans la droite ligne de la modernité, aspire à une autonomie ontologique, c'est-à-dire, à une maîtrise absolue vis-à-vis des processus vivants et donc à une indépendance totale vis-à-vis des contingences et du caractère relativement aléatoire propre au vivant. A tout cela, il aspire sans limite. C'est en cela que ce projet a des fondements métaphysiques (Dupuy 2009:71). En appliquant ces principes, l'agriculture engendre, ô paradoxe, des forces qui la dépassent aboutissant à une perte de maitrise des processus économiques et écologiques qu'elle a, par ailleurs, contribué à mettre en branle. 
Au plan théorique, il peut donc être intéressant de penser ensemble autonomie, hétéronomie et seuils critiques. Sur ces questions, les travaux d'Ivan Illich (1975 et 2006) sont pionniers. Ils ont notamment contribué à la divulgation de la notion de contre-productivité. En nous appuyant sur le résumé qu'en donne Dupuy (1999: 422), rappelons ce qu'il entend par la notion de contre-productivité structurelle des sociétés industrielles pour appliquer son analyse au cas de l'agriculture conventionnelle.

Lorsque le mode hétéronome caractérisant les grands secteurs de la société industrielle (Transport, Santé, Education, Communication), dépasse certains seuils de développement et acquiert un monopole sur d'autres modes de production (mode autonome ${ }^{20}$, autogène, vernaculaire, traditionnel, etc.), celui-ci produit lui-même les obstacles aux objectifs qu'il est censé servir. Ce raisonnement s'applique-t-il à l'agriculture? L'agriculture conventionnelle continue-t-elle d'être une activité nourricière ou est-elle entrée dans une dynamique de destruction (des sols et de la santé)? Nourrit-elle la vie ou la détruit-elle?

On est en droit de se poser la question lorsque, mis bout à bout, on considère les divers effets pervers de celle-ci, en France notamment: stagnation puis perte de la productivité agricole depuis 2009 ne permettant plus à une grande partie des producteurs français de vivre dignement de leur travail ${ }^{21}$, augmentation du mal-être (cf. les centaines de suicides par an ${ }^{22}$, déstructuration des familles, augmentation des maladies chroniques dues à l'alimentation ${ }^{23}$ ). Or, une agriculture qui détruit les sols, empoisonne paysans, riverains et consommateurs, asphyxie les eaux, peut-elle encore être qualifiée de moderne?

Ou bien 'nous n'avons jamais été modernes' (Latour 1991) ou bien la modernité est justement ce projet historique de réinvention permanente capable de questionner les certitudes établies et dans ce cas il devrait être possible de redéfinir ce qu'est une agriculture moderne. Car tous les effets pervers évoqués plus haut, et d'autres encore, nous amènent à nous interroger sur la prétention de l'agricul- 
ture et de l'agronomie conventionnelles à détenir le monopole de la raison scientifique.

Existe-t-il des alternatives ou des solutions à cet état de fait? C'est précisément ce que les divers mouvements s'inscrivant dans une démarche agroécologique cherchent à construire. Mais l'agroécologie offre-t-elle les conditions d'une redéfinition? On peut, en effet, induire un changement social par des pratiques nouvelles mais aussi en élaborant d'autres représentations, de nouveaux discours, de nouvelles définitions de ce que pourrait être une agriculture moderne. C'est ce que nous proposons d'examiner maintenant.

\section{Agroécologie: une autre agriculture pour une nouvelle ère}

Alors même qu'en France l'agriculture conventionnelle passe par une crise de rentabilité, de valeur, d'identité et que son image tend à se détériorer ${ }^{24}$ et que dans certains cas la tentation est de recourir à 'toujours plus de ce qui cause le problème' (plus de subventions pour plus de recours à la chimie et à l'automatisation), la permaculture et l'agroécologie bénéficient, elles, d'une image contemporaine, jeune et positive. La diversité des personnes s'intéressant à la permaculture est très large. Dans les cours auxquels nous avons participé, nous avons identifié des architectes, des agriculteurs, des ingénieurs, des biologistes ou encore des étudiants d'écoles de commerce. Certains s'installent et mettent en place des modèles économiques fondés sur les principes de la permaculture. Dans certains cas, le concept de start-up peut s'appliquer à ces agriculteurs-entrepreneurs récemment installés $^{25}$. Tous ces projets ont en commun de privilégier un développement en réseau devançant parfois le soutien des pouvoirs publics.

Ces exploitations agricoles posent une question aux instituts de recherche agronomique mais plus largement à la mentalité moderne en vigueur, habituée à l'idée qu'une entreprise agricole (ou autre), doive crôttre pour s'enrichir ou simplement survivre. Ces expériences posent la question de l'échelle pertinente et du seuil critique de développement 
au-delà duquel il faut toujours plus de travail et d'énergie pour maintenir productive et rentable l'exploitation, sans l'assurance de réussir.

La permaculture cherche donc à optimiser l'existant en partant des éléments, des fonctions et des besoins/ressources du système local plutôt qu'à maximiser une grandeur de manière isolée et univoque. De même, alors que, comme nous l'avons vu, le marché global impose le rythme de travail et de production jusqu'aux cycles de la plante, certaines approches agroécologiques remettent au goût du jour une certaine autosuffisance (relative, selon les objectifs que se fixe le producteur) permettant les échanges non-marchands entre producteurs et la vente du surplus sur le marché local grâce à un mouvement de relocalisation ${ }^{26}$.

\section{Vers une nouvelle définition de l'agriculture moderne?}

Avec la montée en puissance de l'agroécologie dans ses formes pratiques et théoriques ${ }^{27}$, nous avons affaire à un conflit de définition et de légitimité pour savoir ce qu'est une agriculture moderne. L'un des critères de légitimité pour qu'une agriculture puisse prétendre au qualificatif de moderne est sa capacité à nourrir une population mondiale croissante. Dans le documentaire Voyage entre sols et terre ${ }^{28}$, lorsqu'un agriculteur demande au microbiologiste des sols Dominique Bourguignon ${ }^{29}$ ce qu'il pense du 'bio', il répond:

"Le problème, c'est qu'il n'a pas bénéficié des recherches depuis 50 ans. Cela dit, vous serez tous obligés de faire de l'agriculture biologique dans les années à venir avec des associations complexes et des rotations complexes" (Will, 2011, 37ème minute).

Et à la question 'pourra-t-on nourrir le monde par le bio?', D. Bourguignon dit:

"Oui! Parce que le rendement moyen est de 30 quintaux / ha, à l'heure actuelle sur la planète et on fait 30 quintaux en bio sans problème. On sait qu'on peut parfaitement nourrir l'humanité. Vous savez que les plus gros rendements ne sont pas faits en agriculture conventionnelle. Aucun champ au monde n'est capable de produire autant au mètre carré que le champ des paysans mexicains où il y a le maïs avec le haricot qui s'enroule autour et les courges 
entre les rangs. Le problème c'est qu'on ne sait pas le mécaniser. Mais ne confondez pas productivité par homme et productivité au mètre carré. L'agriculture la plus productive au mètre carré, c'est l'agriculture paysanne. La plus productive par homme, c'est l'intensive. Mais si vous voulez nourrir beaucoup de monde il faudra revenir à une agriculture paysanne. Jamais il n'y a eu autant d'hommes qui ont souffert de la faim qu'en ce moment" (Will, 2011, 37ème minute).

Le Rodale Institute ${ }^{30}$ aux Etats-Unis compilent 30 ans d'études comparatives sur la rentabilité de parcelles cultivées avec des méthodes conventionnelles et avec des méthodes agroécologiques. Les résultats montrent une productivité plus ou moins équivalente en conditions normales et une meilleure résilience des sols agroécologiques lors d'épisodes climatiques extrêmes (sécheresse ou fortes pluies).

\section{L'agroécologie comme conversion?}

Lorsqu'un agriculteur prend conscience de l'impasse dans laquelle il se trouve, il peut être tenté de changer de voie et de modèle, s'il a connaissance que d'autres modèles existent. On parle alors de 'conversion au (ou en) bio' ou de transition agroécologique. Mais de quel ordre cette conversion est-elle? S'agit-il seulement d'une conversion technique, de méthode, d'une opportunité économique? Les motivations sont-elles pragmatiques ou existentielles? Quelles dimensions sont engagées? Cette conversion implique-t-elle un changement de regard, dans la relation de l'agriculteur à la terre?

\section{a - Conditions et dimensions de la conversion}

Parmi tous les aspects évoqués dans les témoignages recueillis lors de nos recherches de terrain, l'expérience existentielle des agriculteurs constitue une motivation importante. Le 'déclic' peut être provoqué par un sentiment de désespoir vis-à-vis d'une terre devenue improductive ou peut être dû à un sentiment de culpabilité. Le témoignage de 
M. Jones, agriculteur agroforestier qui vit à Abreu e Lima, au Pernambouc est, à ce titre, éloquent.

Sa conversion à la production agroforestière correspond à un moment de crise profonde dans sa vie personnelle et professionnelle. Au début des années 1990, Seu Jones a commencé à travailler une terre obtenue à la suite de la réforme agraire située entre les communes d'Abreu e Lima et Igarassu. La terre était très dégradée par la monoculture de canne à sucre et l'agriculteur et sa famille se trouvaient dans une impasse. C'est la rencontre avec le Centro Sabiá en 1994, ONG connue et reconnue dans l'Etat de Pernambouc pour son travail de formation des paysans à des méthodes agroécologiques, qui lui a permis de trouver une issue en l'aidant à se former à l'agroforesterie en mettant en place un SAF (Système Agroforestier). Il a commencé par cesser de 'nettoyer' (portugais: limpar) c'est-à-dire de pratiquer l'abbatis-brûlis systématique sur sa parcelle et de planter de manière 'automatique': riz, maïs, haricots, igname, manioc, etc. Il a commencé par observer et essayer de comprendre comment fonctionne le système faune/flore/sol. Mais, pendant la transition, les 'voisins ont commencé à faire des commentaires':

"Le voisinage parlait mal de moi. Les gens disaient que j'étais devenu fou, que j'avais arrêté de m'occuper de ma terre. Tout simplement parce que j'avais arrêté de couper et brûler mes parcelles avant de planter ce qu'on a toujours planté ici. Pour la première fois, j'observais et j'apprenais comment les éléments du système fonctionnaient ensemble $e^{31}$. Ça a été une période difficile. S'il n'y avait pas eu le Centro Sabiá pour me soutenir, je n'aurais pas tenu le coup" (Seu Jones).

Chez les divers agriculteurs qui témoignent, c'est l'expérience avec la proximité de la mort (maladie ou décès d'un proche par intoxication, épuisement/stérilité de la terre ou plus simplement 'mort' de certitudes) qui constitue un premier pas vers la conversion. La conversion est donc une démarche consciente et globale qui implique toutes les dimensions de l'existence et relève d'un désir d'intégration avec les éléments qui composent le système. Il faut entendre ces agriculteurs 
qui ont opéré la conversion pour voir que cette démarche comble tant les besoins les plus élémentaires que des aspirations plus immatériels.

En effet, en ce qui concerne l'alimentation, les familles que nous avons accompagnées au Brésil à travers l'association Terra e Vida ne craignent pas de manquer de nourriture car le fait de cultiver des espèces de plantes dont le développement est séquentiel (en succession sur toute l'année) fait qu'elles ont constamment de quoi se nourrir, en quantité et variété. Ainsi, elles ne dépendent pas d'une seule récolte. Cela est important car au Brésil la sécurité alimentaire est encore un sujet d'actualité.

Par ailleurs, dans l'approche agroécologique, les dimensions esthétique et poétique sont présentes et satisfont des besoins (ou aspirations) comme l'épanouissement personnel. Les personnes rencontrées éprouvent, en effet, un sentiment d'émerveillement devant l'inventivité de la vie, émerveillement visible dans leurs yeux quand elles évoquent les multiples liens non immédiatement perceptibles qui s'établissent entre tous les éléments du système agroforestier: les insectes, les plantes, les animaux, la terre et les personnes. Cette 'satisfaction existentielle' est encore présente lorsque la démarche agroforestière permet le retour d'une source d'eau jadis disparue du fait d'un traitement inadéquat de la terre ${ }^{32}$.

Enfin, et c'est peut-être l'aspect le plus important car il résume tous les autres, les agriculteurs-rices interviewé.e.s expriment un sentiment de paix qui provient d'une attitude consistant à ne plus être "en guerre contre la terre ni contre autrui" (Seu Jones). Cet élément existentiel est déterminant et nous voudrions conclure sur ce point en évoquant le cas de l'agriculture syntropique, ce qui nous permettra de fermer la boucle avec notre première partie sur les origines sacrificielles.

\section{Le cas de l'agriculture syntropique: une relecture des origines sacrificielles de l'agriculture?}

L'agriculture 'entropique', c'est l'agriculture conventionnelle d'origine sacrificielle mais désacralisée (déritualisée) et qui a cessé, 
dans la modernité, d'être productive par son propre excès de puissance. C'est celle qui continue de recourir à la violence mais sans la modération assurée par les rites. Elle a pu être très productive dans une certaine phase de la modernité tant que les sols étaient encore en bonne santé. Mais si l'on en croit les études mentionnées plus haut, elle a cessé de l'être. En termes anthropologiques, on retrouve l'équivalent de ces paradoxes dans la perte d'efficacité du sacrifice comme trait caractéristique de la modernité. Expliquons-nous.

A l'inverse des sociétés religieuses archaïques qui s'appuient sur le sacrifice (et le rite de manière générale) pour établir ou restaurer, de manière régulière, l'ordre socio-culturel, dans la modernité, les sociétés ne peuvent plus compter sur celui-ci. Selon Girard (1978) cela est dû au fait qu'elles en savent trop sur le caractère ignoble du mécanisme (l'innocence complète ou partielle de la victime) pour adhérer complètement au mythe qui vient légitimer et donner rétrospectivement un sens au sacrifice. Alors que Girard parle de crise sacrificielle, définie comme usure et perte d'efficacité du sacrifice (1972:77), comme dynamique sociologique caractéristique des tragédies grecques, il décrit les sociétés ouvertes, plurielles et complexes contemporaines comme affectée d'une crise sacrificielle démultipliée (Girard, 1978), dans un état de crise permanente qui ne peut trouver de solution définitive (Aubin 2012:229).

Dans les tragédies grecques, chacun des protagonistes assène un coup qui se veut être le dernier mot de la violence. Chaque coup doit faire sortir du désordre et rétablir la hiérarchie, mais c'est l'inverse qui se produit. Chaque coup porté renforce la collaboration négative des jumeaux de la violence, aggravant la crise. Chaque sacrifice qui échoue renforce la spirale de la violence.

Dans le contexte politique contemporain, chaque mesure pour sortir de la crise prétend être la dernière (ce qui conférerait prestige à ses promoteurs) mais ne fait, bien souvent, qu'empirer la crise. La crise de l'agriculture productiviste est peut-être aussi (et avant tout) une crise sacrificielle quand le 'mal nécessaire' (les externalités, les pol- 
lutions, par exemple) ne produit plus de bien mais s'ajoute au mal qu'il est censé éloigner.

Si nous prenons l'exemple de l'agriculture syntropique ${ }^{33}$, une branche de l'agroécologie, il semble s'agir d'un véritable retournement de la logique sacrificielle en reprenant la question à la source: le sol ${ }^{34}$. Un système syntropique, c'est un système capable d'enrichir le sol, d'accumuler de la diversité et de l'énergie au cours du temps ${ }^{35}$. La nature cesse alors d'être perçue comme l'ennemi et devient une alliée. Mieux: un modèle à imiter, un professeur. Ce changement d'attitude (cette conversion) est illustré par l'usage du terme cova qui, en portugais, signifie aussi bien la fosse où l'on enterre le mort que le trou où l'on jette la graine. Ce double sens, nous l'avons exploré dans la première partie. Prenant acte (au moins implicitement) de la dimension sacrificielle et morbide associée au terme cova, les praticiens de l'agriculture syntropique choisissent d'utiliser le terme berço (berceau) pour désigner le trou où seront déposées les graines.

Par ailleurs, dans le débat qui voit s'opposer les doctrines conservationniste/préservationnistes de la nature à l'agriculture intensive/ conventionnelle/productiviste, l'agriculture syntropique renvoie dos à dos ces deux ensembles d'acteurs. Les deux, selon elle, se justifiant l'un l'autre en une collaboration négative. Si l'on ne peut toucher à une nature sanctuarisée et, à certains égards, mythifiée (vierge, pure, exempte de toute intervention), alors il devient nécessaire de 'sacrifier' des terres pour produire de manière intensive. L'agriculture syntropique dépasse l'opposition en étant capable de faire cohabiter des plantes (annuelles et pérennes) dont le développement est séquentiel et qui occupent toutes les strates (sol, bas, moyen, haut et émergent) associant ainsi la présence de la forêt et celle de plantes comestibles. Enfin, le tour de force de l'agriculture syntropique est d'avoir introduit les concepts d'amour inconditionnel et d'abondance ${ }^{36}$ à la base de la méthode:

"L'amour inconditionnel, c'est le don de soi dont font preuve les plantes pour un tout qui leur est supérieur. L'abondance, c'est le résultat de cette relation vertueuse." ${ }^{\text {7 }}$ 
Il fallait faire preuve d'un certain courage de la part d'Ernst Götsch pour introduire ces concepts en agronomie alors que l'agronomie conventionnelle est indexée au paradigme économique dominant qui postule que les ressources sont rares et que les rivalités, médiées par le marché, produisent l'abondance, gage de paix sociale. Dupuy et Dumouchel (1979) ont bien montré que ce sont précisément les rivalités mimétiques qui produisent la rareté! L'agriculture syntropique (ainsi que d'autres approches agroécologiques) apporte la preuve expérimentale que considérer la nature comme généreuse et abondante et imiter ce postulat (en faire un modèle) en élaborant une méthode et une démarche à partir de cela, peut produire, de fait, de l'abondance ${ }^{38}$.

Avec l'agriculture syntropique, nous avons donc l'exemple remarquable d'un paradigme de transition d'un modèle sacrificiel de l'autre (c'est-à-dire d'éléments hors système absorbés puis rejetés) à un modèle sacrificiel de soi (amour inconditionnel générateur d'abondance). Il s'agit à notre avis d'une véritable conversion au sens défini plus haut.

Que des besoins alimentaires, économiques, sociaux (par les relations d'entraide ou celles établies sur les marchés locaux) intellectuels (stratégies nouvelles à trouver pour résoudre les problèmes qui en permaculture contiennent leur propre solution), esthétiques, existentiels voire spirituels (provenant du sentiment d'intégration avec la terre) puissent être satisfaits par une même approche agroécologique, cela interroge et défie les paradigmes dominants qui semblent, eux, à bout de souffle. Les recherches sont à poursuivre pour relativiser, si besoin, un constat qui pourrait paraitre idyllique, recenser les expériences qui fonctionnent et celles qui fonctionnent moins bien. En tout cas, il s'agit, nous semble-t-il, d'une tentative de nouvelle alliance société/ nature/agriculture.

\section{Conclusion}

Nous avons choisi de présenter un panorama de l'agriculture de ses origines sacrificielles (hypothétiques) et rituelles jusqu'à ses formes 
contemporaines: agriculture conventionnelle et agroécologie. Alors que dans les sociétés traditionnelles le rapport à la terre est chose sérieuse et grave et que la consommation des aliments est elle-même un enjeu considérable impliquant des forces cosmiques et sociales intégrées en un tout cohérent, dans les sociétés modernes, les dimensions sociales et symboliques demeurent, certes, (c'est un postulat sociologique élémentaire) mais l'enjeu alimentaire se réduit souvent à des questions de prix, de nutrition, de calories (en excès ou manquantes), à un enjeu de santé publique et de bien-être individuel. Le rapport sacré à la terre et à ses produits induisaient des limites qui restreignaient le pouvoir de l'agir humain sur le monde. Dans la modernité, ce qui guide l'action humaine est uniquement fondé sur la rationalité instrumentale, par une adéquation des moyens à mobiliser en vue d'une fin plus grande. La terre devient un moyen de produire pour une finalité qui peut être éthique, politique (nourrir la population) et économique. Cette finalité étant jugée plus élevée justifie la pollution, la 'violence' faite à la terre. On voit que la rationalité instrumentale garde les traces de la logique sacrificielle (Dupuy 2009) en s'appuyant sur le raisonnement 'la fin justifie les moyens', en ce qu'elle serait une dose de violence, 'un mal', pour un Bien supérieur.

Mais ce que l'on découvre aujourd'hui, c'est que lorsque la rationalité instrumentale, livrée à elle-même, sans limite, produit les conditions de dépassement de certains seuils critiques inscrits dans la structure même de tous systèmes vivants (sociétés humaines ou écosystèmes naturels), la Raison produit le contraire de ce qu'elle est censée produire: s'auto-empêche (devient de moins en moins efficace et rentable) et grignote le sens (tant la direction à suivre que la dimension symbolique de l'institution). C'est la contre-productivité structurelle illichienne appliquée à l'agriculture caractéritisque d'une situation globale de saturation propre à l'anthropocène.

Si l'agriculture a des origines sacrificielles, si le labour, les semailles et les moissons ont des origines rituelles, alors nous soutenons que l'agroécologie s'inscrit dans une démarche authentiquement mo- 
derne de critique et de relecture plus globale et fondamentale de la logique sacrificielle. Cette relecture critique et subversive provient du constat que le sacrifice (mal nécessaire pour un bien plus grand) qui pouvait encore fonctionner parce que reconnu comme légitime par les grands récits religieux, puis politiques, dans les divers domaines de la société, ne fonctionne plus, y compris dans le champ de l'agriculture. L'agroécologie serait alors le renoncement à recourir à un remède qui ne guérit plus. Pire, qui rend malade...

Pour être un objet pluriel (mouvement social, science, ensemble de pratiques) articulant les savoirs anciens et contemporains et montrant l'impossibilité sociale, écologique et symbolique de poursuivre dans la voie actuellement privilégiée, l'agroécologie fournit-elle les bases d'une refondation de la relation humains/non-humains prenant acte de l'obsolescence des catégories qui nous ont jusqu'alors guidés et qu'il nous faut désormais entièrement repenser à l'aune de la nouvelle situation planétaire: l'anthropocène? Nous n'avons fait qu'esquisser des pistes de réflexion qu'il faudra poursuivre tant le chantier est immense.

\section{Notes}

1 Nous utilisons ici le mode conditionnel car le terme n'est pas encore officiellement accepté par la commission internationale de stratigraphie, l'un des organes de l'Union Internationale des Sciences Géologique (IUGS). Il s'agit d'un processus scientifique rigoureux et long au cours duquel on évalue si les activités anthropiques ont laissé des marques significatives dans la structure géologique de la planète.

2 Quelles dates doit-on prendre en considération? Si l'on part du principe que, à diverses époques de son histoire l'homme s'est peu à peu émancipé des cycles saisonniers et naturels jusqu'à en modifier la composition et la structure: le contrôle des techniques du feu (-400.000 ans), la 'Révolution néolithique' (-10.000 ans), la Révolution Industrielle du $19{ }^{\text {ème }}$ siècle ou encore l'invention et l'utilisation de la bombe thermonucléaire (1945). Crutzen est partisan de cette dernière date et de la Grande Accélération d'après-guerre (Steffen et al. 2015) pour dater l'origine de la phase II de l'Anthropocène.

3 Nous remercions vivement Lucien Scubla d'avoir partagé avec nous ses réflexions sur le sacrifice (communication privée). 
4 Rapporté par Brandenburger (1931:34-35).

5 Un tour d'horizon de la mythologie mondiale nous a permis de recenser le mythe de Milomaki des Indiens Yahuna (Amazonie Colombienne) recueilli par Theodor Koch-Grünberg et analysé par Girard (1978:120 -121) dans lequel Milomaki est tué et incinéré et des cendres duquel naît le palmier Paschiuba. Il existe aussi le mythe des indiens Zuni sur l'origine de la plante datura inoxia rapporté par Hofmann \& Schultes (2005:106); le mythe gabonais sur l'origine de la plante Iboga, rapporté par Hofmann \& Schultes (2005:113). Nous avons également recensé um mythe cherokee (Yoshida 1966, 724) et péruvien (Yoshida 1966, 727) sur l'origine du maïs. Voir encore le mythe d'Uke Moshi au Japon, rapportant l'apparition du riz et autres plantes à la base de l'alimentation nippone à partir du corps de la victime enterrée. (Yoshida 1966, 719). Voir également celui d'Hainuwele (Ile de Céram, Mélanésie) rapporté par Alfred Jensen (cité par Yoshida 1966, 726). Enfin, parmi les mythes brésiliens on trouve celui d'Onhiámuáçabê sur l'origine du guaraná qui naît de l'oeil enterré de la victime (Pereira 1954). Le meurtre fondateur structure également les mythes de Purusha dans l'Inde antique, de Pan'Ku en Chine, d'Ymir en Scandinavie et on retrouve aussi le thème du corps démembré dans une infinité d'autres mythes duquel surgissent l'univers ou, plus modestement, les plantes comestibles. Le thème du démembrement du corps rappelle également le sparagmos dionysiaque. Voir également le récit mythique du mithraïsme.

6 Sans s'attaquer aux 'vraies' causes, bien entendu. Celles-ci demeurent inatteignables en la personne de la seule victime dans le cas de la rivalité ou dans le cas d'un événement climatique, par exemple.

7 La victime sacrificielle est une victime de substitution à la victime émissaire, ellemême 'première' victime se substituant à la foule (Girard 1972).

8 Cité par Goblet d'Alviella dans Les rites de la moisson et les origines de l'agriculture (1898:10-11).

9 C'est aussi l'idée avancée par des archéologues, notamment par Smith (1995:80). Par ailleurs, en Albanie, lors de conflits liés à la terre, un duel était organisé selon le code coutumier consigné dans le Kanun. Là où tombait le corps de l'un des deux rivaux, la stèle marquait le champ. La foule, témoin du duel, était garante du respect de la limite du champ. Quiconque s'emploierait à mettre en cause la limite subirait le même sort que le vaincu. Voir Ismael Kadaré, Auril Brisé, Le Livre de Poche, Paris, 1983.

10 C'est nous qui soulignons.

11 Eck, Bernard, Le pharmakos et le meurtrier, in V. Liard (éd.), Histoires de crimes et société, EUD, Dijon, 2011:15-29. Aux îles Fidji, M. Fison a remarqué "dans les enclos sacrés d'abondantes récoltes d'ignames, engendrées par les offrandes de provisions aux ancêtres. Des faits analogues ont été rapportés à Madagascar, dans l'Afrique Centrale et dans l'archipel Malais" (Alviella 1898:12). Et d'ajouter: "Comme, inévitablement, les effets bénéfiques du sacrifice s'estompent, il faut alors répéter l'opération chaque année à la saison des semailles en immolant et en enfouissant une victime humaine qui deviendra ainsi l'esprit de la prochaine récolte. Des exemples sont donnés chez les Pawnies des Etats-Unis sacrifiaient chaque année une jeune fille dans la période des semailles, chez les Cafres, du Betchuanaland" (Alviella 1898:14-15). 
12 Nous nous appuyons essentiellement sur la recension qu'en a faite Geneviève Calame-Griaule (Calame-Griaule 1962).

13 On note à ce propos, à titre d'illustration, que le portugais cova désigne à la fois la fosse où l'on enterre le mort et le trou où l'on dépose les graines. Nous y reviendrons dans la troisième partie.

14 Pour plus de détails sur les implications de ce paradoxe et une proposition de résolution, voir Dupuy 2009.

15 Après que le christianisme soit devenu religion officielle de l'Empire sous Constantin en 312.

16 Voir Michel Serres (2008:21).

17 Sur les effets corrosifs du christianisme sur le sacré, voir, entre autres, Illich (2006).

18 L'exemple fameux des Nuer décrit par Evans-Pritchard (1973) montre qu'ils cohabitaient avec leur bétail qui constituait une société double en miroir de la leur. Les vaches portaient des noms, l'organisation était hiérarchisée. Sacrifier une vache c'était un peu sacrifier un homme. Le bétail constituait donc une réserve de victimes sacrificielles à 'portée de mains'.

19 Cf. Rapport Stern: http://webarchive.nationalarchives.gov.uk/+/http:// www.hm-treasury.gov.uk/d/stern_longsummary_french.pdf ou encore le Rapport Rocktröm http://www.nature.com/nature/journal/v461/n7263/full/461472a. html.

20 Le mode autonome n'implique pas nécessairement l'autosuffisance de l'unité de production. Il implique un mode de production qui ne dépende entièrement pas du marché et des institutions spécialisées dont les biens et les services sont dispensés par des experts ou des secteurs spécialisés (privés ou publics) moyennant transactions monétaires prises en compte par la comptabilité nationale. Voir Illich 1975.

21 En France, la perte des sols (en plus d'autres facteurs) provoque une stagnation de la productivité agricole. Voir 'L'agriculture et l'agroalimentaire français à la recherche d'une compétitivité perdue' ISS-Inra Sciences Sociales - Recherches em Economie et Sociologie Rurales, N 4-5/2011 - Février 2012, disponible sur le site http://www.tse-fr.eu/sites/default/files/medias/doc/Publications/Vincent_Requillart/iss11_45_1.pdf ou encore http://www.lesechos.fr/10/01/2016/lesechos. fr/021608908597_la-fertilite-des-sols-part-en-poussiere.htm.

22 Il existe une controverse sur les chiffres concernant le nombre de suicides dans la profession agricole. L'INVS avance des chiffres contestés par les associations de familles de victimes de suicides. Pour plus de détails consulter: http://invs. santepubliquefrance.fr/fr/Publications-et-outils/Rapports-et-syntheses/Travail-etsante/2016/Surveillance-de-la-mortalite-par-suicide-des-agriculteurs-exploitants, puis http://arsfp.blogspot.com.br/.

23 Voir le rapport de l'OMS établissant une corrélation entre alimentation et maladies chroniques: http://www.who.int/nutrition/publications/obesity/WHO_ TRS_916/fr/.

24 Voir le sondage: http://www.ifop.com/?option=com_publication $\&$ type $=$ poll\&id=2943. 
Ce sont généralement des petites entreprises, très dynamiques et visibles sur Internet. Voir, par exemple, les fermes de la Goursaline (http://www.permaculturedesign.fr/), de la Bourdaisière (http://www.fermesdavenir.org/), du Bec-Hellouin qui obtiennent des rendements élevés sur des surfaces relativement petites travaillées en bio-intensif. Voir à ce propos l'étude menée par l'Inra http://www.fermedubec.com/inra/Rapport-étude-2011-2015-Bec-Hellouin_30112015-2.pdf. Des expériences agroécologiques existent aussi sur des surfaces immenses comme la Fazenda da Toca au Brésil (2300 hectares dans l'Etat de São Paulo). Pour plus de détails sur cette approche voir le documentaire présenté à la COP21: Life in Syntropy accessible ici: https://www.youtube.com/watch?v=gSPNRu4ZPvE.

26 Sur les questions de la sécurité et de la souveraineté alimentaires, ceci s'avère déterminant comme nous avons pu le constater au Brésil. Voir l'exemple rapporté dans le point III-2.

27 Le Rapport d'Olivier de Schutter, publié en 2011 lorsqu'il était Rapporteur spécial pour le droit à l'alimentation au Conseil des droits de l'homme à l'ONU affirme que seule l'agroécologie peut nourrir le monde. Ce rapport a contribué à donner une légitimité accrue à l'agroécologie dans le débat avec l'agriculture conventionnelle.

28 Médecins de l'Humus? Un Voyage entre Sols et Terre (54 mn), un film de Jean Will.

29 Dominique Bourguignon et son épouse, Lydia, ont créé en 1991 le LAMS, un laboratoire spécialisé dans l'analyse de la microbiologie des sols.

30 Le rapport est disponible à l'adresse suivante: http://rodaleinstitute.org/ourwork/farming-systems-trial/farming-systems-trial-30-year-report/.

31 C'est nous qui soulignons.

32 Ce dont nous avons pu être témoin dans une petite ferme à Igarassu (dans le Pernambouc).

33 L'agriculture syntropique est une méthode agroécologique élaborée par le chercheur et agroforestier suisse Ernst Götsch. Pour plus d'informations: http://agendagotsch.com/pt/syntropy.

34 Dans les courants agroécologiques, il est courant d'entendre que humain et humus proviennent de la même racine. Maurice Tournier nous apprend que l'affaire n'est pas si simple mais que ce n'est pas à exclure. http://www.persee.fr/doc/ mots_0243-6450_2001_num_65_1_2494.

35 Nous avons eu l'occasion de suivre un cours d'agriculture syntropique au Sitio Semente près de Brasilia du 9 au 12 mars 2017. Les résultats en termes de productivité défient les modèles utilisés par les techniciens de l'EMBRAPA (l'équivalent de l'INRA au Brésil).

36 Termes fréquemment employés dans les cours et les écrits relatifs à l'agriculture syntropique. Par exemple: "Dans la nature, il n'existe pas de compétition froide. Toutes les relations sont basées sur la coopération et l'amour inconditionnel, toujours orientées vers la réalisation d'une fonction”. Source: http://agendagotsch. $\mathrm{com} / \mathrm{pt} / \mathrm{ernst}$.

37 Propos de Juã Pereira, formateur em agriculteur syntropique, recueillis lors de la formation évoquée plus haut. 
Les premières mesures chiffrées de la productivité réalisées par un agronome de l'Université de Brasilia font état de plusieurs dizaines de tonnes par hectare et par an. Voir http://repositorio.unb.br/bitstream/10482/14683/1/2013_MauricioRigonHoffmannMoura.pdf.

\section{Reférences}

ALVIELLA, Goblet d'. 1898. Les Rites de la Moisson et les Commencements de l'agriculture. Paris: Ernest Leroux.

AUBIN, Ludovic. 2012. Paradoxes, apories et contradictions au coeur du paradigme du développement durable. Etude d'un champ institutionnel de la région métropolitaine de Recife et de la zone de la Forêt Atlantique de Pernambouc, Brésil. Lille: ANRT.

BECK, Ulrich. 2001. La Société du Risque: Sur la voie d'une autre modernité. Paris: Aubier.

BONANNO, A. \& CAVALCANTI, J. S. B. 2014. Labor Relations In Globalized Food: Research in Rural Sociology and Development. Bingley: Emerald Group Publishing Limited.

BONNEUIL Christophe. 2013. L'Événement Anthropocène: La Terre, l'histoire et nous. Paris: Seuil.

BRANDENBURGER, Clemente. 1931. Lendas dos Nossos Índios: leituras brasileiras. Rio de Janeiro: F. Alves.

CALAME-GRIAULE, Geneviève. 1963. "Jean SERVIER. Les portes de l'année. Rites et Symboles. L’Algérie dans la tradition méditerranéenne.”Journal de la Société des Africanistes, 33(2):315-317.

CAUVIN, Jacques. 1994. Naissance des Divinités, Naissance de l'Agriculture. La révolution des symboles au néolithique. Paris: C.N.R.S.

CAVALCANTI, Josefa S. B. 2003. "El 'medio ambiente' como objeto de las Ciencias Sociales: un análisis basado en los estudios de globalización de los sistemas agroalimentarios”. In CAVALCANTI, J. S. B. et al (eds.): El Campo en la Sociología actual: una perspectiva latinoamericana, pp. 157-176. Buenos Aires: La Colmena.

2013. "Comunidades Rurais e os desafios da sociedade contemporânea". In SILVA, W. A. \& CARMO, R. M. (eds.): Mundo Rural: Mito ou realidade?, pp. 67-82. São Paulo: Annablume.

CAVALCANTI, J. S. B. \& BENDINI, M. I. 2014. "Globalization and change in labor relations in fruit regions of Brazil and Argentina”. In BONANNO, A. \& CAVALCANTI, J. S. B. (eds.): Research in Rural Sociology and Development, pp. 3-32. Bingley: Emerald Group Publishing Limited.

CRUTZEN, P. \& STOERMER, E. 2000. “The 'Anthropocene”, Global Change, NewsLetter, 41:17-18.

DUMOUCHEL, P. \& DUPUY, J.-P. 1979. Le Signe et l'Envie. Paris: Seuil. 
DUPUY, Jean-Pierre. 2009. La Marque du Sacré. Paris: Carnets Nord. . 1999. Éthique et Philosophie de l'Action. Paris: Ellipses.

ECK, Bernard. 2011. "Le pharmakos et le meurtrier". In LIARD, V. (ed.): Histoires de crimes et société, pp. 15-29. Dijon: EUD.

ELIADE, Mircea. 2006. L'Épreuve du Labyrinthe. Entretiens avec Claude-Henri Rocquet. Paris: Editions du Rocher.

EVANS-PRITCHARD, Edward. 1973. Parenté et Mariage chez les Nuer. Paris: Payot. GIRARD, René. 1972. La Violence et le Sacré. Paris: Grasset. . 1978. Des Choses Cachées Depuis la Fondation du Monde. Paris: Grasset. 2004. Aux Origines de la Culture. Paris: Desclée de Brouwer.

HOCART, Arthur. 2005. Au Commencement Était le Rite. Paris: La Découverte/ M.A.U.S.S.

HOFMANN, A. \& SCHUlTES, R. E. 2005. Les Plantes des Dieux: Les plantes hallucinogènes. Botanique et ethnologie. Paris: Edition du Lézard.

ILLICH, Ivan. 1975. Némésis Médicale. Paris: Seuil. . 2006. La Corruption du Meilleur Engendre le Pire. Arles: Actes Sud.

KADARE, Ismaïl. 1983. Avril Brisé. Paris: Le Livre de Poche.

LATOUR, Bruno. 1991. Nous n'Avons Jamais Été Modernes - Essai d'anthropologie symétrique. Paris: La Découverte.

MOURA, Maurício R. H. 2013. Sistemas Agroflorestais para Agricultura Familiar: Análise econômica. Brasília: UNB. (http://repositorio.unb.br/ bitstream/10482/14683/1/2013_MauricioRigonHoffmannMoura.pdf; accédé le 08/01/2016)

PEREIRA, Nunes. 1954. Os Índios Maués. Rio de Janeiro: Organização Simões.

ROCKSTRÖM, Johan et al. 2009. "A safe operating space for humanity". Nature, 461:472-475. (www.nature.com/nature/journal/v461/n7263/full/461472a. html; accédé le 13/02/2016).

ROSA, Hartmut. 2013. Accélération, Une Critique Sociale du Temps. Paris: La Découverte.

SERRES, Michel. 1993. La Légende des Anges. Paris: Flammarion.

SERVIER, Jean. 1951. "Les rites du labour en Algérie."Journal de la Société des Africanistes, 21(2):175-196.

. 1962. Les Portes de l'Année. Rites et symboles. L'Algérie dans la tradition méditerranéenne. Paris: Robert Laffont.

SMITH, Bruch. 1995. The Emergence of Agriculture. New York: Scientific American Library.

YOSHIDA, Atsuhiko. 1966. "Les excrétions de la Déesse et l'origine de l'agriculture". Annales, Économies, Sociétés, Civilisations, 21(4):717-728. 


\section{Autres Documents}

AGROPARISTECH et INRA. 2015. Etude "Maraîchage 'biologique' 'permaculturel' et 'performance' 'économique”, novembre 2015. (www.fermedubec.com/ inra/Rapport-étude-2011-2015-Bec-Hellouin_30112015-2.pdf; accédé le 17/10/2016).

INSEE. 2013. Tableaux de l'Économie Française. (www.insee.fr/fr/themes/ document.asp?ref_id=T13F172; accédé le 20/01/2016).

KHIREDDINE-MEDOUNI, Imane. 2016. Surveillance de la mortalité par suicide des agriculteurs exploitants. Situation 2010-2011 et évolution 2007-2011. (http://invs.santepubliquefrance.fr/fr/Publications-et-outils/Rapports-etsyntheses/Travail-et-sante/2016/Surveillance-de-la-mortalite-par-suicide-desagriculteurs-exploitants; accédé le 20/02/2017).

MANN, Charles. 2011. “The Birth of Religion”. National Geographic. (http:// ngm.nationalgeographic.com/2011/06/gobekli-tepe/mann-text; accédé le 12/03/2016).

MOLGA, Paul. 2016. "La fertilité des sols part en poussière". Les Echos. (www. lesechos.fr/10/01/2016/lesechos.fr/021608908597_la-fertilite-des-sols-parten-poussiere.htm; accédé le 12/02/2017).

MONOCULTURA do Eucalipto e suas Implicações. 2012. (http://monografias. brasilescola.uol.com.br/geografia/a-monocultura-eucalipto-suas-implicacoes. htm; accédé le 05/01/2016).

RÉQUILLART, Vincent. 2012. ISS, Recherches en Economie et Sociologie Rurales.

(www.tse-fr.eu/sites/default/files/medias/doc/Publications/Vincent_Requillart/ iss11_45_1.pdf; accédé le 15/04/2017).

RODALE INSTITUTE. Rapport d'activités. (http://rodaleinstitute.org/ourwork/farming-systems-trial/farming-systems-trial-30-year-report/; accédé le 05/02/2016).

STERN, Nicholas. 2007. The Economics of Climate Change: the Stern review. Cambridge, UK: Cambridge University Press.

OMS. 2003. Rapport d'une Consultation OMS/FAO d'experts (Rapport technique de l'OMS 916). (www.who.int/nutrition/publications/obesity/WHO_ TRS_916/fr/; accédé le 19/03/2017).

\section{Documents Audio-Visuels}

ROBIN, Marie-Monique. 2012. Les Moissons du futur. Arte Éditions.

WILL, Jean. 2016. Médecins de l'Humus? Un Voyage entre Sol et Terre - Soigneurs de terres. (54 mn.). Diffusé sur France 2 le 31/01/2016. 
Resumo: Este artigo apresenta a hipótese de uma origem ritual da agricultura. Os autores situam a agricultura convencional e a agroecologia em uma perspectiva histórica, na qual a modernidade apresenta a característica de ser um processo de dessacralização do Homem em relação ao mundo de forma geral e à terra e ao solo de forma específica. A dessacralização é considerada aqui como um processo de superação ou transgressão de todos os limites simbólicos que outrora continham a poder do agir do ser humano no mundo. Essa ação hoje se tornou tão sistemática e profunda que o debate existe para saber se a humanidade entrou em uma nova era geológica: o antropoceno. Será a agroecologia o único paradigma capaz de enfrentar os desafios dessa nova era?

Palavras-chave: Antropologia; Sociologia da Agricultura; Agroecologia; Antropoceno.

\begin{abstract}
This paper asserts a ritual-based origin hypothesis for agriculture. The authors situate conventional agriculture and agroecology in a broad historical perspective, throughout which modernity is described as a process of secularization of the relationship of Man with the world and especially with earth and soil. Secularization is then defined as a process of transgression of any symbolical limits that once contained the power of human action onto the world. In modern times, this action turned itself so deep and systematic that a new concept arose to describe our era: Anthropocene. Is agroecology the only paradigm able to lead with the challenges of this brand new period?
\end{abstract}

Keywords: Anthropology; Sociology of Agriculture; Agroecology; Anthropocene.

Recebido em julho de 2017.

Aprovado em agosto de 2017. 